\title{
Do Grandchildren whose Grandmother Smoked while Pregnant with the Mother have a Higher Risk of Childhood Asthma? A Protocol for a Systematic Review.
}

Catherine J Fleming ( $\boldsymbol{\sigma}$ cfleming27@qub.ac.uk)

Queen's University Belfast https://orcid.org/0000-0001-5789-3389

Jonathan P Fleming

Queen's University Belfast

Judith R Fleming

NHS Lanarkshire

Dara O'Donoghue

Queen's University Belfast

Michael D Shields

Queen's University Belfast

\section{Protocol}

Keywords: Child, asthma, recurrent wheeze, grandmaternal smoking, transgenerational.

Posted Date: July 2nd, 2021

DOI: https://doi.org/10.21203/rs.3.rs-657126/v1

License: (c) (i) This work is licensed under a Creative Commons Attribution 4.0 International License.

Read Full License 


\section{Abstract}

Background: Mothers smoking while pregnant is known to increase the offspring risk of subsequent wheezing or asthma. It is less well known whether this phenomenal occurs trans-generationally, which would suggest potential epigenetic transfer. If a link is identified, this would strengthen the need for antismoking strategies during pregnancy and would direct further research towards understanding the epigenetic mechanisms. We aim to perform a systematic review and meta-analysis to determine whether or not grandchildren of grandmothers who smoked while pregnant with their mother have a higher risk of childhood wheeze or asthma.

Methods: The systematic review will use the Preferred Reporting items for Systematic Review and MetaAnalysis Protocols (PRISMA-P) checklist. To identify relevant studies, we will search databases including Medline, PubMed, Embase, Web of Science and Scopus.

The exposure being investigated is whether the maternal grandmother smoked in pregnancy and the outcome being measured is recurrent wheeze and/or asthma in the grandchild.

Studies will be screened according to inclusion and exclusion criteria to include primary cohort studies of any study design type. Eligible studies will then be evaluated for quality and assessed for bias independently by three reviewers using the "Grading of Recommendations, Assessment, Development and Evaluation" (GRADE) approach. The results of the studies will be extracted into 2x2 outcome tables and a meta-analysis will be carried out. The statistical software used will be StatsDirect.

Discussion: This review will help determine whether there is transgenerational inheritance of wheeze and asthma, beyond the risk of direct in utero exposure.

Systematic Review Registration: PROSPERO REG: CRD42021234346

\section{Background}

Smoking during pregnancy is known to be associated with the development of asthma and recurrent wheeze in the child [1]. Some recent studies have also observed that grandmaternal smoking while pregnant with the mother may increase the risk of the grandchild developing asthma, irrespective of the mother's smoking habits. The transgenerational inheritance of asthma phenotypes, mediated by epigenetic changes across the germline, has been clearly demonstrated in animal models [2]. It is not currently known whether there is similar transgenerational transfer of asthma in humans, and the data from various cohort studies is conflicting $[3,4]$.

Asthma is the most prevalent chronic respiratory disease worldwide [5] and it's prevalence continues to increase [6]. It is a key public health issue, costing the UK $£ 1.1$ billion in 2011 [7]. Asthma is known to have a range of environmental, genetic and host risk factors [8,9]. Recently, epigenetics is garnering interest as a key component of asthma aetiology, since neither genetics nor environment consistently 
account for the range of asthma phenotypes [10]. Epigenetics refers to heritable modifications of gene expression without changes to the DNA sequence and is often induced in response to environmental exposures. It is known that prenatal smoking in mothers leads to widespread changes in patterns of foetal DNA methylation [11] which are more pronounced than those caused by environmental tobacco smoke (ETS) exposure after birth [12]. As such epigenetic changes are heritable, it is plausible that a grandchild's asthma could be linked to grandmaternal smoking when the mother is in utero.

Robust data is lacking regarding grandparent environmental exposures affecting grandchild disease [13]. A systematic review and meta-analysis are necessary to collate the data from disparate cohort studies, thereby providing strength to the observation and eliminating any chance findings. An established association between grandchild asthma and maternal grandmother smoking during pregnancy would be strongly suggestive of epigenetic transfer. This would highlight an area for further research. Indeed, there is a recent trend in allergic disease research towards the analysis of epigenetic modifications, enabled by several large-scale epigenomic-wide association studies (EWAS) [14]. Epigenetic modifications constitute a novel class of biomarker and therefore warrant investigation as potential new therapeutic targets [15]. Additionally, if grandmother smoking habits are shown to increase the grandchild's asthma risk, this may aid understanding of the recent rise in asthma and provide further justification for the prevention of smoking during pregnancy.

A number of studies have highlighted gendered inheritance patterns, although these are also conflicting $[4,16]$. This systematic review will thus also consider gender subgroups in order to eliminate chance findings. Knowledge of sex specific effects is a valuable pointer in discovering the timing, location and mechanisms of epimutations. Gender differences in the inheritance of recurrent wheeze versus asthma could indicate different epigenetic aetiologies for the two connected conditions.

\section{Methods/design}

This systematic review and meta-analysis will investigate if grandmother smoking when pregnant with the mother increases the risk of the grandchild developing asthma or recurrent wheeze. A literature search will be carried out across a number of databases, including Medline, Embase, Web of Science, PubMed, Scopus, Cochrane Library, Global Health using a comprehensive search strategy. Inclusion and exclusion criteria are summarised in Table 1 and an example search strategy is shown in Table 2. Additional references will be identified by searching the references cited in eligible studies.

Studies will be screened according to title and abstract, and then the text body, using clear inclusion and exclusion criteria. This protocol follows the Preferred Reporting items for Systematic Review and MetaAnalysis Protocols (PRISMA-P) recommendations. The Preferred Reporting Items for Systematic Review and Meta-Analysis (PRISMA) standards will be adhered to in reporting the findings (Additional File I: Appendix I) [17].

\section{Inclusion/exclusion criteria}


Table 1

Inclusion and Exclusion criteria.

Include:

Cohort studies

Primary studies, including peer-reviewed and grey literature

Publication available in English

All ethnicities

Population: grandchildren

Exposure: Maternal grandmother smoking when mother in utero

Comparison: Asthma/wheeze risk in grandchild of maternal grandmothers who smoked during pregnancy, versus maternal grandmothers who did not.
Exclude:

Reviews

Letters

Case Reports

Unpublished cohort data that has not been extracted

Population: co-morbidities; family history of asthma.

Exposure: Mother smoking when child in utero; Mother smoking ex utero; Grandmother smoking ex utero; ETS.

Comparison: Asthma/wheeze risk in child of mother who smoked during pregnancy; risk from ETS.

Outcome: Recurrent wheeze or asthma development in grandchild

Table 2 outlines the inclusion and exclusion criteria used when screening first by title and abstract and then by full text. Papers will be included only if they are of cohort study design. The population being studied must be grandchildren diagnosed with asthma and/or recurrent wheeze. The studies must be investigating grandmother smoking during pregnancy with the mother, compared with not smoking, on the outcome of grandchild recurrent wheeze or asthma. No studies investigating a population of grandchildren with comorbidities will be included, and no other asthma risk factors will be considered.

\section{Types of studies and participants}

This review will include cohort studies only. Participant data including grandchild age, asthma and wheeze diagnoses, and grandmother and mother smoking statuses will depend on the specific data recorded in each cohort study. Participants will be considered in line with the inclusion criteria.

\section{Exposure, comparison and outcome}

The exposure being investigated is maternal grandmother smoking when the mother was in utero. This is being compared against no smoking. The outcome being measured is the odds ratio of grandchild asthma and/or recurrent wheeze.

\section{Information source and search strategy}

The literature search will be carried out electronically using a strategy developed in collaboration with the Queens University Belfast Medical Librarian. To ensure all potential literature is included, we will search 
Embase, Medline, PubMed, Web of Science and the Cochrane Library. An example of the planned electronic search strategy including limits applied can be seen in Table 2 .

Table 2

Search strategy.

\begin{tabular}{|c|c|c|}
\hline \# & Searches & Results \\
\hline 1 & exp Tobacco Smoking/ or smoking.mp. or Smoking/ & 286093 \\
\hline 2 & Asthma/ or asthma.mp. & 176414 \\
\hline 3 & allergy.mp. or Hypersensitivity/ & 108266 \\
\hline 4 & $\begin{array}{l}\text { (recurrent adj2 wheeze).mp. [mp = title, abstract, original title, name of substance } \\
\text { word, subject heading word, floating sub-heading word, keyword heading word, } \\
\text { organism supplementary concept word, protocol supplementary concept word, rare } \\
\text { disease supplementary concept word, unique identifier, synonyms] }\end{array}$ & 364 \\
\hline 5 & 2 or 3 or 4 & 264467 \\
\hline 6 & grandmaternal.mp & 108 \\
\hline 7 & grandmother.mp & 1821 \\
\hline 8 & 6 or 7 & 1902 \\
\hline 9 & Intergenerational Relations/ or Grandparents/ & 4236 \\
\hline 10 & grandparent*.mp. & 3423 \\
\hline 11 & $\begin{array}{l}\text { (transgenerational adj2 relations).mp. [mp = title, abstract, original title, name of } \\
\text { substance word, subject heading word, floating sub-heading word, keyword heading } \\
\text { word, organism supplementary concept word, protocol supplementary concept word, } \\
\text { rare disease supplementary concept word, unique identifier, synonyms] }\end{array}$ & 1 \\
\hline 12 & $\begin{array}{l}\text { (multigenerational adj2 relations).mp. [mp = title, abstract, original title, name of } \\
\text { substance word, subject heading word, floating sub-heading word, keyword heading } \\
\text { word, organism supplementary concept word, protocol supplementary concept word, } \\
\text { rare disease supplementary concept word, unique identifier, synonyms] }\end{array}$ & 2 \\
\hline 13 & 9 or 10 or 11 or 12 & 6887 \\
\hline 14 & 1 and 2 and 8 & 9 \\
\hline 15 & 1 and 5 and 8 & 9 \\
\hline 16 & 8 or 13 & 8484 \\
\hline 17 & 1 and 2 and 16 & 19 \\
\hline 18 & 1 and 5 and 16 & 19 \\
\hline
\end{tabular}

Table 2 is an example of the comprehensive literature search which will be carried out across all the electronic databases, with search terms and limitations applied. This search strategy example is from MEDLINE. 


\section{Data collection and analysis \\ Selection of studies}

The studies will be independently screened according to the inclusion and exclusion criteria by two reviewers. The screening will be a two-step process, first by title and abstract, and then by full-text. Those excluded by full text will be listed and explained in the appendix of the final report. A third-party reviewer will be involved in the case of any disagreements. Using a reference software (EndNote), any duplicate articles will be identified. Any relevant reviews found in the literature search will only be used to source additional primary studies for this review.

\section{Data extraction and management}

We will use the standard Population, Exposure, Comparison and Outcome (PECO) approach. The population is defined as grandchildren of grandmothers who smoked while pregnant. The exposure is grandmother smoking when the mother is in utero. The primary outcome is recurrent wheeze and asthma development in the grandchild. Data will be extracted using an adapted form from the Data Collection check list (Cochrane Collaboration) [18]. An example of this is in Additional file 1: Appendix 2.

Data will be presented in a summary of findings table including the types of studies, population number, number in exposure group, comparison groups, $2 \times 2$ outcome results tables, odds ratios and a column for the evaluation of the quality of evidence and bias risk. This summary of findings table will be presented in the results section, as per the Cochrane handbook. If any data is missing, we will contact the authors of the paper to obtain the complete set.

\section{Risk of bias}

The risk of bias and quality of evidence will be evaluated independently by three reviewers using the "Grading of Recommendations Assessment, Development and Evaluation" (GRADE) approach. This grades the evidence as being of high, moderate, low or very low quality by using the study design as a starting point and upgrading or downgrading the evidence according to certain criteria. Five factors which lower the quality of the evidence include limitations of study design and execution leading to bias, inconsistency or heterogeneity, indirectness, imprecision and publication bias. The most likely bias is reporting bias.

\section{Outcomes}

To ensure comparability between studies, the primary outcome being investigated for this review is any recurrent wheeze or asthma, including parent-reported wheeze as well as doctor-diagnosed wheeze or asthma. It is important to include parent-reported wheeze as not all children who wheeze will be assessed by a physician. Individual studies may measure other relevant outcomes such as atopy, hypersensitivity and allergy. However, these are not a priority for this review. They have been included in the search strategy to ensure all citations of asthma and recurrent wheeze are caught in the literature search. 


\section{Data synthesis and meta-analysis}

Using the main outcome of asthma (dichotomous-yes/no) and the data from the $2 \times 2$ outcome tables produced, a meta-analysis will be performed using a random-effects model, with odds ratio as the principal summary measure. Individual studies will be represented on a forest plot based on odds ratios and $95 \%$ confidence intervals. Funnel plots will also be generated to portray publication bias or possible selective reporting within studies. The software which will be used for the meta-analysis is StatsDirect version 3.3 (2020) [19]. Sub-group analysis will be carried out in grandchildren whose mother also smoked during pregnancy and in grandchildren whose paternal grandmother smoked during pregnancy, in order to compare their risk of asthma/recurrent wheeze with those whose sole exposure was from the maternal grandmother's in utero smoking. This will provide insight into where the greatest risk lies in terms of in utero smoking and will help elucidate possible pathways and mechanisms of intergenerational asthma inheritance.

\section{Heterogeneity}

To test for heterogeneity (inconsistency between studies), we will use the $\mathrm{I}^{2}$ test, taking an $\mathrm{I}^{2}$ of $>75 \%$ as being high heterogeneity. Sub-group analysis looking at grandchildren whose paternal grandmother smoked during pregnancy may be carried out to explore the possible transmission of epigenetic asthma inheritance via the male germ line.

\section{Safety}

There are no safety concerns.

\section{Discussion}

This review will help determine whether there is transgenerational inheritance of wheeze and asthma, beyond the risk of direct in utero exposure. A positive finding will strengthen the argument against maternal smoking and open the door to further research into the inheritance mechanisms.

\section{List Of Abbreviations}

PRISMA-P (Preferred Reporting items for Systematic Review and Meta-Analysis Protocols)

GRADE (Grading of Recommendations, Assessment, Development and Evaluation)

EWAS (Epigenomic-wide Association Studies)

ETS (Environmental Tobacco Smoke)

PECO (Population, Exposure, Comparison, Outcome)

\section{Declarations}




\section{Contributions}

CJF and MDS conceived study and drew up first draft protocol. JPF, JRF and DO'D reviewed the first draft protocol and provided feedback for improvement. CJF, MDS, JPF, JRF and DO'D will all be involved with data extraction, interpretation and final report writing. MDS is the guarantor of this study and results.

\section{Financial Support}

There is no specific funding for this project.

\section{Availability of data}

The minimal dataset will be placed on the Queen's University Belfast data repository. The data will be made available upon reasonable request.

\section{References}

1. Burke $\mathrm{H}$, et al. Prenatal and Passive Smoke Exposure and Incidence of Asthma and Wheeze: Systematic Review and Meta-analysis. Pediatrics. 2012;129(4):735-44.

2. Rehan VK, et al. Perinatal nicotine exposure induces asthma in second generation offspring. BMC Med. 2012;10(1):129.

3. Li Y-F, et al. Maternal and Grandmaternal Smoking Patterns Are Associated With Early Childhood Asthma. Chest. 2005;127(4):1232-41.

4. Miller LL, et al. Is the Growth of the Fetus of a Non-Smoking Mother Influenced by the Smoking of Either Grandmother while Pregnant? PLOS ONE. 2014;9(2):e86781.

5. Collaborators GBDCRD. Global, regional, and national deaths, prevalence, disability-adjusted life years, and years lived with disability for chronic obstructive pulmonary disease and asthma, 19902015: a systematic analysis for the Global Burden of Disease Study 2015. The Lancet Respiratory medicine. 2017;5(9):691-706.

6. Loftus PA, Wise SK. Epidemiology of asthma. Curr Opin Otolaryngol Head Neck Surg. 2016;24(3):245-9.

7. Mukherjee $\mathrm{M}$, et al. The epidemiology, healthcare and societal burden and costs of asthma in the UK and its member nations: analyses of standalone and linked national databases. BMC Med. 2016;14(1):113.

8. Eder W, Ege MJ, von Mutius E. The Asthma Epidemic. N Engl J Med. 2006;355(21):2226-35.

9. Martinez FD, Vercelli D. Asthma The Lancet. 2013;382(9901):1360-72.

10. Vercelli D. Does epigenetics play a role in human asthma? Allergology International. 2016;65(2):1236.

11. Joubert BR, et al. DNA Methylation in Newborns and Maternal Smoking in Pregnancy: Genome-wide Consortium Meta-analysis. Am J Hum Genet. 2016;98(4):680-96. 
12. Rauschert S, et al. Maternal Smoking During Pregnancy Induces Persistent Epigenetic Changes Into Adolescence, Independent of Postnatal Smoke Exposure and Is Associated With Cardiometabolic Risk. Frontiers in genetics. 2019;10:770-0.

13. Arshad SH, et al. Multigenerational cohorts in patients with asthma and allergy. J Allergy Clin Immunol. 2017;139(2):415-21.

14. Kabesch $M$, Tost J. Recent findings in the genetics and epigenetics of asthma and allergy. Semin Immunopathol. 2020;42(1):43-60.

15. Tost J. A translational perspective on epigenetics in allergic diseases. J Allergy Clin Immunol. 2018;142(3):715-26.

16. Lodge CJ, et al. Grandmaternal smoking increases asthma risk in grandchildren: A nationwide Swedish cohort. Clinical Experimental Allergy. 2018;48(2):167-74.

17. Moher D, Liberati A, Tetzlaff J, Altman DG, The PRISMAG. Preferred Reporting Items for Systematic Reviews and Meta-Analyses: The PRISMA Statement. PLoS Med. 2009;6(7):e1000097. doi:10.1371/journal.pmed1000097.

18. Higgins JPT, Thomas J, Chandler J, Cumpston M, Li T, Page MJ, Welch VA, editors. Cochrane Handbook for Systematic Reviews of Interventions version 6.1 (updated September 2020). Cochrane, 2020. Available from .

19. StatsDirect Ltd. StatsDirect statistical software. http://statsdirect.com. England. Stats Ditrect Ltd; 2020.

\section{Supplementary Files}

This is a list of supplementary files associated with this preprint. Click to download.

- Appendix1and2.docx 